Fetal Diagnosis and Therapy
Fetal Diagn Ther 2019;46:166-174

DOI: $10.1159 / 000495506$
Received: July 13, 2017

Accepted after revision: November 14, 2018

Published online: January 10, 2019

\title{
Differences in Origin and Outcome of Intra-Abdominal Cysts in Male and Female Fetuses
}

\author{
Marjolein Husen ${ }^{a}$ Pauline C. Schut ${ }^{a}$ Adriana C.H. Neven ${ }^{a}$ \\ Nagma Yousoufi ${ }^{a}$ Nanko de Graaf ${ }^{b}$ Cornelius E.J.Sloots ${ }^{c}$ Alex J. Eggink ${ }^{a}$ \\ Titia E. Cohen-Overbeek ${ }^{a}$ \\ ${ }^{a}$ Erasmus MC, University Medical Center Rotterdam, Department of Obstetrics and Gynecology, Division of \\ Obstetrics and Prenatal Medicine, Rotterdam, The Netherlands; ${ }^{b}$ Erasmus MC, University Medical Center Rotterdam, \\ Department of Pediatric Radiology, Rotterdam, The Netherlands; ' Erasmus MC, Sophia Children's Hospital, \\ University Medical Center Rotterdam, Department of Pediatric Surgery, Rotterdam, The Netherlands
}

\section{Keywords}

Intra-abdominal cyst · Ovarian cyst · Fetal gender .

Anorectal malformation

\begin{abstract}
Objective: To investigate the origin and outcome in a cohort of male and female fetuses with intra-abdominal cysts, in order to provide recommendations on management and to improve prenatal counselling. Methods: From 2002 to 2016, intra-abdominal cysts were detected by ultrasound in 158 fetuses. Cases with an umbilical vein varix were excluded. Fetal, neonatal, and maternal characteristics were retrieved from electronic patient files. Results: In female fetuses ( $n=$ 114), intra-abdominal cysts were diagnosed at a later gestational age compared with male fetuses $(n=44)$ (median 32.0 vs. 21.5 weeks, $p<0.001$ ). The maximum prenatal cyst diameter was larger in female fetuses (median 35 vs. $17 \mathrm{~mm}, p<$ $0.001)$. Associated anomalies were less frequent in females ( $n=15,13.2 \%$ ) compared with males ( $n=15,34.1 \%)$. In females ( $n=114)$, most cysts were of ovarian origin $(n=81$, $71.1 \%)$. Surgery was performed in 30 (26.3\%) female and 15 (34.1\%) male neonates $(p=0.33)$. Anorectal malformations
\end{abstract}

\begin{tabular}{ll}
\hline KARGER & ( 2019 The Author(s) Karger \\
& Published by S. Karger AG, Basel Open access \\
E-Mail karger@karger.com & This article is licensed under the Creative Commons Attribution- \\
www.karger.com/fdt & $\begin{array}{l}\text { NonCommercial-NoDerivatives 4.0 International License (CC BY- } \\
\text { NC-ND) (http://www.karger.com/Services/OpenAccessLicense). } \\
\text { Usage and distribution for commercial purposes as well as any dis- } \\
\text { tribution of modified material requires written permission. }\end{array}$
\end{tabular}

were present in 6 cases and often not recognized prenatally. Conclusions: The differences in the origin of intra-abdominal cysts between male and female fetuses, resulting in differences in prenatal presentation and postnatal outcome should be taken into account in prenatal counseling within a multidisciplinary team. Evaluation of the fetal perianal muscular complex is indicated.

(c) 2019 The Author(s)

Published by S. Karger AG, Basel

\section{Introduction}

An intra-abdominal cyst is a relatively common finding on ultrasound during pregnancy and is most frequently detected in the second or third trimester [1-4]. Cysts that appear in the first trimester are rare and when isolated, the outcome is usually good [5]. During the development of intra-abdominal structures, cystic formation may occur and cysts mainly originate from either the gastrointestinal tract or the genitourinary tract [6]. They can resolve spontaneously without any clinical conse-

M.H. and P.C.S. contributed equally to this work.
Pauline C. Schut

Erasmus MC, University Medical Center Rotterdam

Department of Obstetrics and Gynecology, Division of Obstetrics and Prenatal Medicine Room Na-1523, Postbus 2040, NL-3000 CA Rotterdam (The Netherlands)

E-Mail p.schut@erasmusmc.nl 
quences or may be a sign of an abnormality that requires close prenatal surveillance and/or surgical intervention after birth $[1-3,7]$. It remains a challenge to make a correct diagnosis prenatally $[1,4]$, which is essential both for optimal counselling of the future parents and for the prenatal and postnatal management.

Previous studies on fetal intra-abdominal cysts have not differentiated between gender, and the majority of studies concentrated on ovarian cysts, as these are relatively common $[8,9]$. Due to the different etiology of intra-abdominal cysts in male and female fetuses, gender should be taken into account in a differential diagnosis.

The primary aim of this study was to investigate the origin and outcome in a cohort of both male and female fetuses with intra-abdominal cysts of uncertain origin, in order to provide recommendations on management and to improve prenatal counselling. Secondarily, the accuracy of prenatal diagnoses and rates of surgery was determined.

\section{Patients and Methods}

Fetuses diagnosed with an intra-abdominal cyst between 2002 and 2016 were selected from the ultrasound database (Astraia Software $\mathrm{GmbH}$, Germany) of the Department of Obstetrics and Gynecology, Erasmus MC, University Medical Center Rotterdam, The Netherlands. All ultrasound examinations were performed by fetal medicine specialists with experience in prenatal medicine using a Voluson E8 System (GE Healthcare, Milwaukee, WI, USA) or a HTL HDI 3000 (Philips Healthcare, Eindhoven, The Netherlands). Cases with a cyst most likely of gastrointestinal origin were included, but cases with an obvious intestinal obstruction or cystic kidney dysplasia were excluded.

Maternal, fetal, and neonatal characteristics were retrieved from the electronic patient records. Histological reports after surgery or autopsy were recorded. A cyst was defined as complex when debris, multiple septations, or echogenicities/calcifications were seen during at least one of the ultrasound examinations [10]. Polyhydramnios was defined as an amniotic fluid index $>24 \mathrm{~cm}$. Delivery before a gestational age of 37 weeks was considered preterm. In the presence of multiple anomalies and/or suspicion of increased risk for chromosomal anomalies, invasive prenatal testing was offered.

All cases with cysts $\geq 40 \mathrm{~mm}$ or associated abnormalities were discussed in a multidisciplinary meeting with prenatal medicine specialists, obstetricians, pediatric surgeons, and neonatologists to determine the prenatal and postnatal management. Prenatal aspiration of large (suspected ovarian) cysts is not recommended in our hospital. Neonates were examined by a pediatrician within $24 \mathrm{~h}$ after birth. If cysts had resolved prenatally, postnatal ultrasound was not routinely performed. In cases with persistent cysts, a transabdominal ultrasound examination was made within the first 2 weeks after birth. If cysts were not detectable in an asymptomatic neonate or had resolved postnatally, no further follow-up was planned. In neonates with persistent cysts, postnatal diagnosis was made using clinical examination by pediatrician and/or pediatric surgeon. Imaging was performed using ultrasound or other methods of imaging on indication. In neonates with complex ovarian cysts, tumor markers were determined.

Final diagnosis was based on postnatal histology report, surgical findings, or results of radiographical studies. If the final postnatal diagnosis had been included in the differential diagnosis prenatally, prenatal diagnosis was considered "correct." In female fetuses, cysts that were diagnosed in the third trimester and had resolved prenatally or postnatally were regarded as ovarian cysts, provided that this was also considered the most likely prenatal diagnosis based on the localization and ultrasound characteristics. Neonates with major congenital abnormalities were included in a standardized follow-up program [11].

SPSS Statistics (released 2013, IBM SPSS Statistics for Windows, version 21.0., IBM Corp., Armonk, NY, USA) was used for statistical analyses. Determination of differences between groups was calculated using independent sample $t$ tests for normally distributed continuous data, Mann-Whitney $U$ test for non-normally distributed continuous data, and $\chi^{2}$ test and Fisher's exact test for categorical data. Descriptive statistics were used to evaluate outcome parameters. A $p$ value $<0.05$ was considered statistically significant. This study was approved by the institutional ethics committee (MEC-2016-539).

\section{Results}

In the study period, 158 fetuses were diagnosed with an intra-abdominal cyst. The study population consisted of more female $(n=114,72.2 \%)$ than male fetuses $(n=$ $44,27.8 \%$ ). Table 1 shows the maternal, pregnancy, and cyst characteristics, categorized by gender. The median maximum prenatal diameter of the cyst was significantly larger in females compared to males ( 35 vs. $17 \mathrm{~mm}, p<$ $0.001)$. If ovarian cysts were excluded $(n=81)$, the maximum prenatal diameter still significantly differed between males and females (median $23 \mathrm{~mm}, p=0.04$ ), but the differences in gestational age at diagnosis (25.6 weeks, range $12.1-38.1, p=0.15$ ) and associated anomalies (prenatally $9 / 33$ vs. $15 / 44, p=0.52$; postnatally $6 / 33$ vs. $9 / 44$, $p=0.39$ ) between males and females became non-significant. Despite prenatal regression of the cyst, abnormalities were present after birth in 1 female and 2 males $(1 / 12$ [8.3\%] vs. $2 / 8$ [25.0\%], $p=0.54)$. These were all anorectal malformations. In one of these cases, the ultrasound report described non-visualization of the fetal anus.

Prenatal chromosomal analysis was performed in 10 female fetuses; genetic abnormalities were found in 3 (30.0\%). One fetus with an intra-abdominal cyst and associated abnormalities was diagnosed with trisomy 21. An 8p23.1 microdeletion was found in a fetus with a cardiac abnormality and a splenic cyst and a $16 \mathrm{p}$ deletion was found in a fetus with multiple severe anomalies. The $16 p$ 
Table 1. Overview of maternal, cyst, and pregnancy characteristics, according to gender

\begin{tabular}{|c|c|c|c|}
\hline Total study population $(n=158)$ & Male fetuses $(n=44)$ & Female fetuses $(n=114)$ & $p$ value \\
\hline Maternal age, years & $31(19-46)$ & $30(19-43)$ & 0.12 \\
\hline Primigravida & $11(25.0)$ & $35(30.7)$ & 0.48 \\
\hline \multicolumn{4}{|l|}{ Conception } \\
\hline Spontaneous & $42(95.5)$ & $97(85.1)$ & 0.54 \\
\hline ICSI & 0 & $3(2.6)$ & \\
\hline IVF & 0 & $2(1.8)$ & \\
\hline Other & 0 & $5(4.4)$ & \\
\hline Unknown & $2(4.5)$ & $7(6.1)$ & \\
\hline GA at time of diagnosis, weeks & $21.5(10.6-36.7)$ & $32.0(12.1-38.3)$ & $<0.001$ \\
\hline Ovarian cyst & & $32.8(23.9-38.3)$ & $<0.001$ \\
\hline Non-ovarian cyst & & $25.6(12.1-38.1)$ & 0.15 \\
\hline Polyhydramnios & $1(2.3)$ & $6(5.3)$ & 0.67 \\
\hline Maximum prenatal diameter of the cyst, $\mathrm{mm}$ & $17(4-94)$ & $35(5-85)$ & $<0.001$ \\
\hline Ovarian cyst & & $38(11-85)$ & $<0.001$ \\
\hline Non-ovarian cyst & & $23(5-76)$ & 0.04 \\
\hline \multicolumn{4}{|l|}{ Characteristics of the cysts } \\
\hline Simple & $33(75.0)$ & $73(64.0)$ & 0.19 \\
\hline Associated anomalies prenatally & $15(34.1)$ & $15(13.2)$ & 0.003 \\
\hline Associated anomalies postnatally & $10(22.7)$ & $11(9.6)$ & 0.005 \\
\hline Spontaneous prenatal regression & $8(18.2)$ & $12(10.5)$ & 0.20 \\
\hline GA at time of diagnosis, weeks & $16.8(10.6-21.1)$ & $30.1(12.1-34.9)$ & 0.02 \\
\hline Maximum prenatal diameter of the cyst, $\mathrm{mm}$ & $10(4-17)$ & $23(6-43)$ & 0.05 \\
\hline Pregnancy outcome & & & 0.24 \\
\hline Live born & $42(95.5)$ & $113(99.1)$ & \\
\hline Neonatal death & $2(4.5)$ & $1(0.9)$ & \\
\hline IUFD & $1(2.3)$ & 0 & \\
\hline TOP & $1(2.3)$ & $1(0.9)$ & \\
\hline GA at birth*, weeks & $38.9(30.3-41.3)$ & $39.0(32.9-41.6)$ & 0.28 \\
\hline Delivery $<37$ weeks* & $6(14.3)$ & $15(13.3)$ & 0.87 \\
\hline Birth weight, $g^{*}$ & $3,510(1,670-4,875)$ & $3,315(1,275-4,510)$ & 0.38 \\
\hline Duration of follow-up, weeks* & $38.8(0-630.1)$ & $24.6(0.1-537.1)$ & 0.41 \\
\hline
\end{tabular}

Data presented as median and range or number and percentage. ICSI, intracytoplasmic sperm injection; IVF, in vitro fertilization; GA, gestational age; IUFD, intrauterine fetal death; TOP, termination of pregnancy. * Excluding IUFD and TOP.

deletion was not considered as being causative for the anomalies. In 13 male fetuses, prenatal chromosomal analysis was performed; in one fetus, a 15q11.2q13.1 deletion with Prader-Willi syndrome was detected.

\section{Cysts Diagnosed in the First Trimester}

First-trimester cysts were seen in 2 female fetuses (1.8\%) and 3 male fetuses (6.8\%). In both females the cysts resolved spontaneously during pregnancy and no abnormalities that could be related to the abdominal cyst were detected after birth. In all 3 male fetuses, the cysts resolved in the second trimester. Two of these male fetuses appeared to have an anorectal malformation at birth.

\section{Cysts Diagnosed in the Second/Third Trimester}

The fetal and neonatal outcome is shown in more detail in a separate flowchart for females and males (Fig. 1 and 2 , respectively). In female fetuses, most cysts $(n=112$, 98.2\%) were detected in the second or third trimester. All females with an isolated cyst and 12/14 (85.7\%) with associated abnormalities were born alive. One neonatal death occurred. This neonate had multiple anomalies and died shortly after birth due to pulmonary hypoplasia. The cyst resolved prenatally, but the anal sphincter could not be visualized and anorectal malformation was suspected. After birth, in addition to associated anomalies, the suspected anorectal malformation was confirmed. One pregnancy was terminated because of trisomy 21 and multiple 


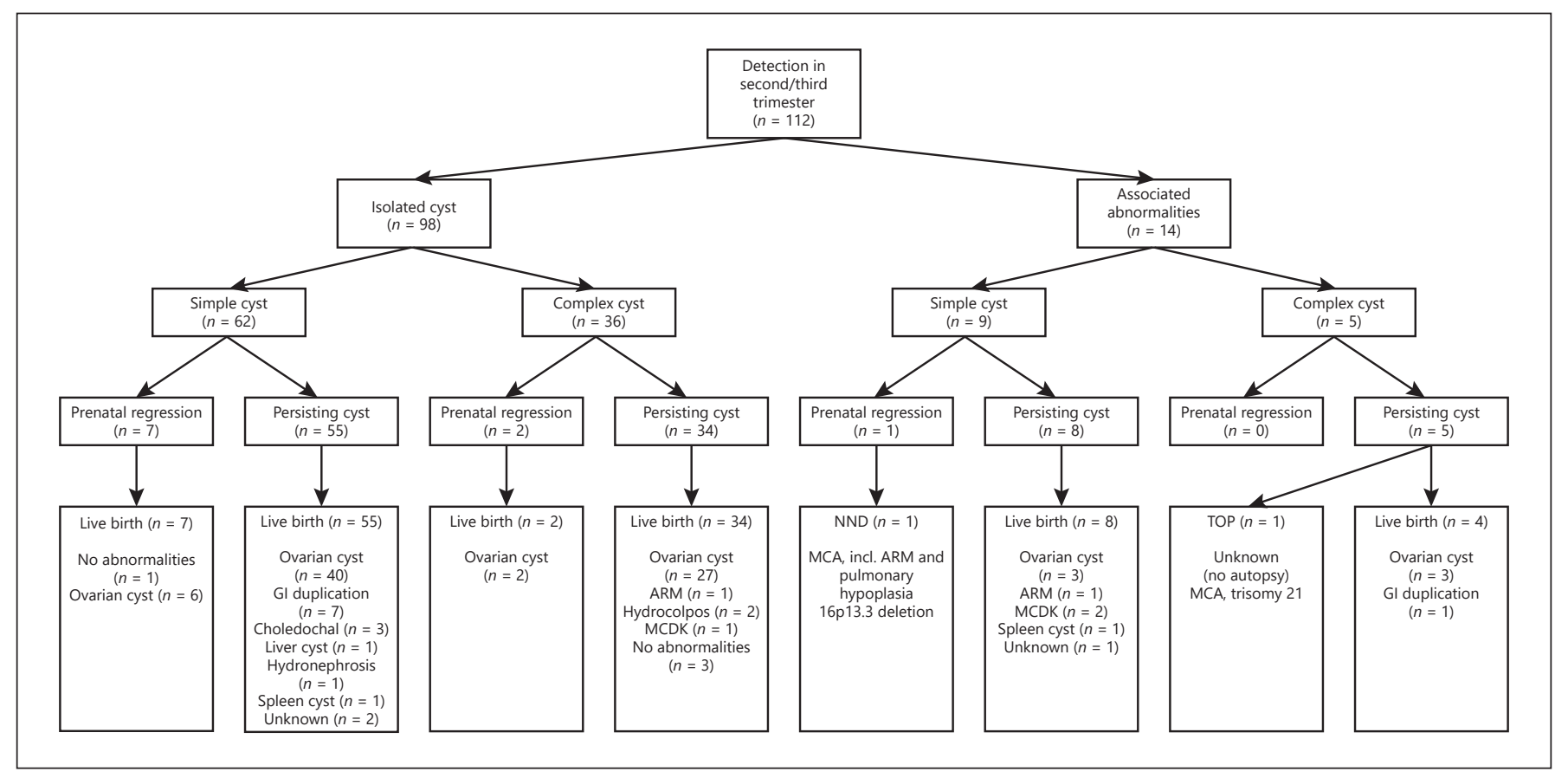

Fig. 1. The outcome of female fetuses diagnosed with an intra-abdominal cyst in the second and third trimester. TOP, termination of pregnancy; NND, neonatal death; ARM, anorectal malformation; GI, gastrointestinal; MCDK, multicystic dysplastic kidney; MCA, multiple congenital abnormalities.

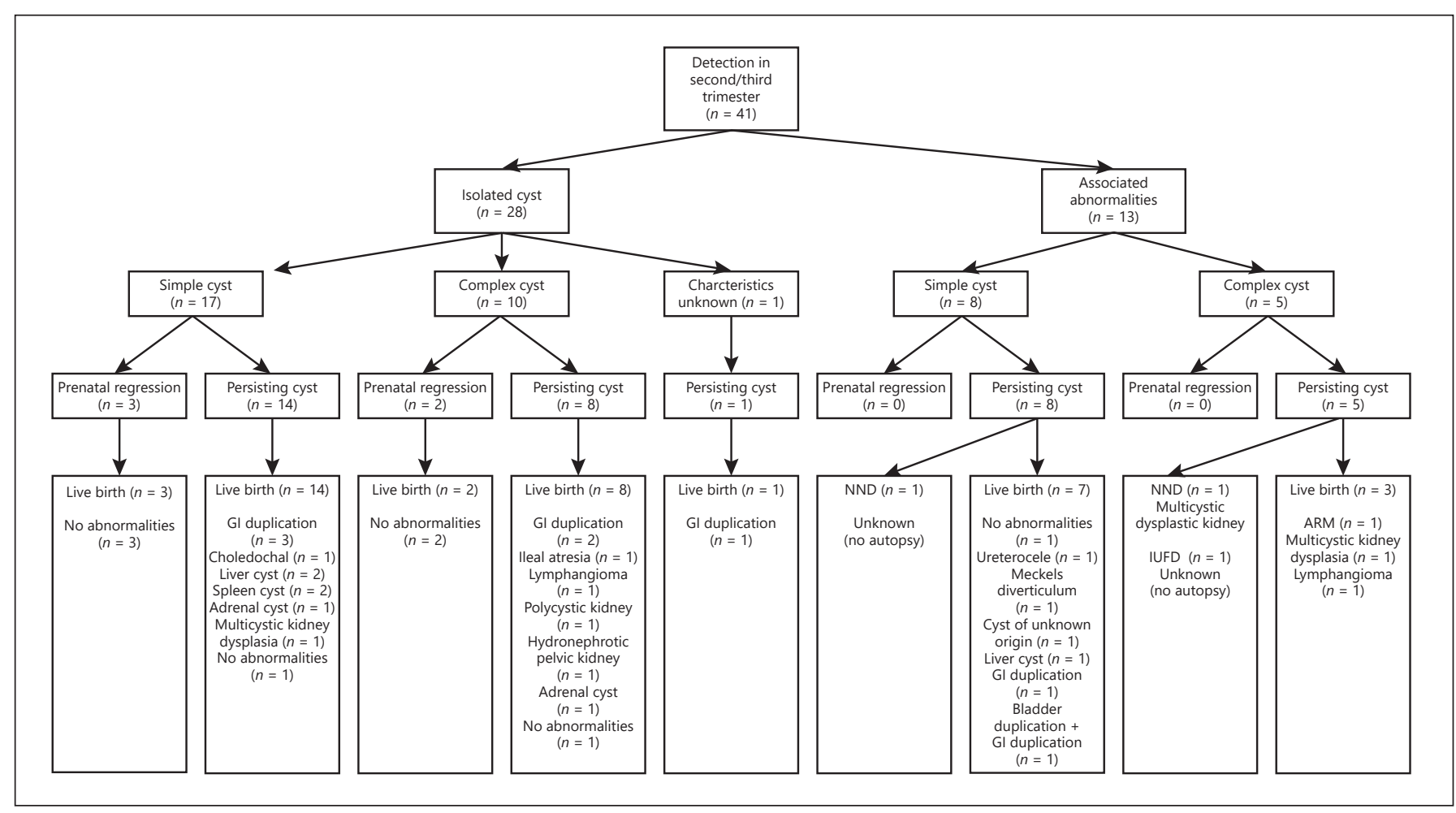

Fig. 2. The outcome of male fetuses diagnosed with an intra-abdominal cyst in the second and third trimester. NND, neonatal death; IUFD, intrauterine fetal death; ARM, anorectal malformation; GI, gastrointestinal. 


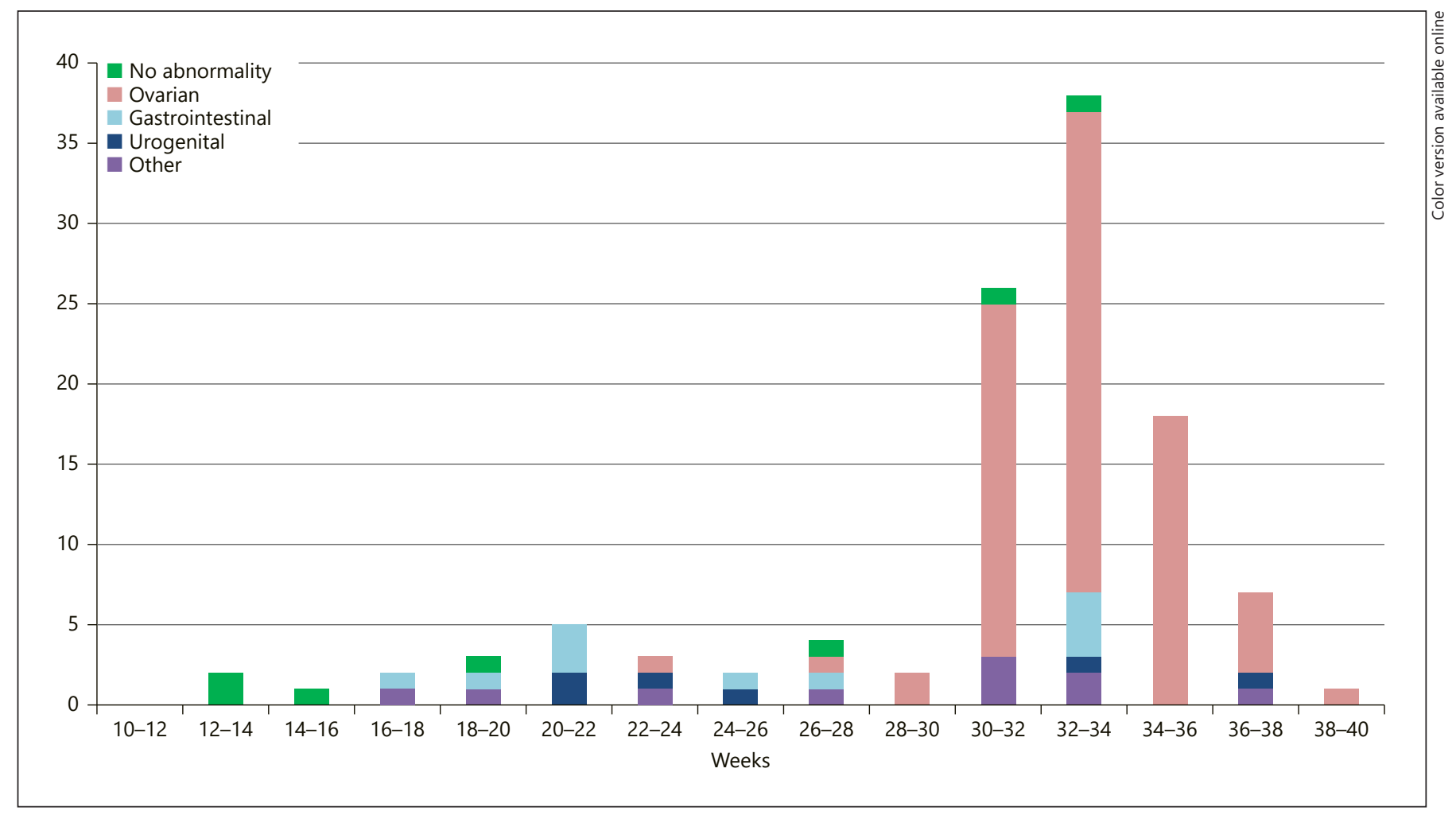

Fig. 3. The distribution of postnatal diagnoses according to gestational age at diagnosis in female fetuses.

associated anomalies. The origin of the cyst remained unknown, as autopsy was not performed.

In male fetuses, the majority of cysts was detected in the second or third trimester $(n=41,93.2 \%)$. All males with an isolated cyst $(n=28,68.3 \%)$ were born alive. In 18 of $23(78.3 \%)$ fetuses with a persisting isolated cyst, abnormalities were present. The majority of males with associated abnormalities and persisting cysts (12/13, 92.3\%) was born alive, but one intrauterine fetal demise occurred in a fetus with multiple intra-abdominal cysts, who was suspected to have cutis laxa syndrome. Two neonatal deaths occurred. One had a displaced unilateral multicystic kidney and multiple other anomalies, including an anorectal malformation (related to VACTER-L association). In the other case, the origin of the intra-abdominal cyst remained unknown since autopsy was not performed.

The division of the postnatal diagnoses according to gestational age at diagnosis and gender is shown in Figure 3 and 4.

\section{Postnatal Outcome}

After birth, an intra-abdominal cyst or a structural anomaly likely related to the prenatally detected intraabdominal cyst was diagnosed in 50 female neonates (43.9\%) and in 30 male neonates (68.2\%). No malignancies were found. An overview of the postnatal diagnoses is provided in Table 2. The associated anomalies in the 5 cases with an ovarian cyst were a double aortic arch, a unilateral multicystic dysplastic kidney, unilateral hydronephrosis, unilateral renal agenesis, and bilateral inguinal hernia.

In the majority of male neonates, intra-abdominal cysts were an isolated finding after birth $(n=21)$, but in 9 neonates $(20.5 \%)$ associated abnormalities were present. These mainly occurred in cases with an anorectal malformation $(n=3)$ and in cases with cystic kidneys $(n=3)$.

The rate of surgery between males and females was not significantly different (15/44 vs. 30/114, $p=0.33$ ) (Table 2 ). Surgery was performed in approximately one-quarter of the female neonates when a cyst was seen prenatally $(n=30,26.3 \%)$, but this proportion was higher $(n=30 / 50$, 


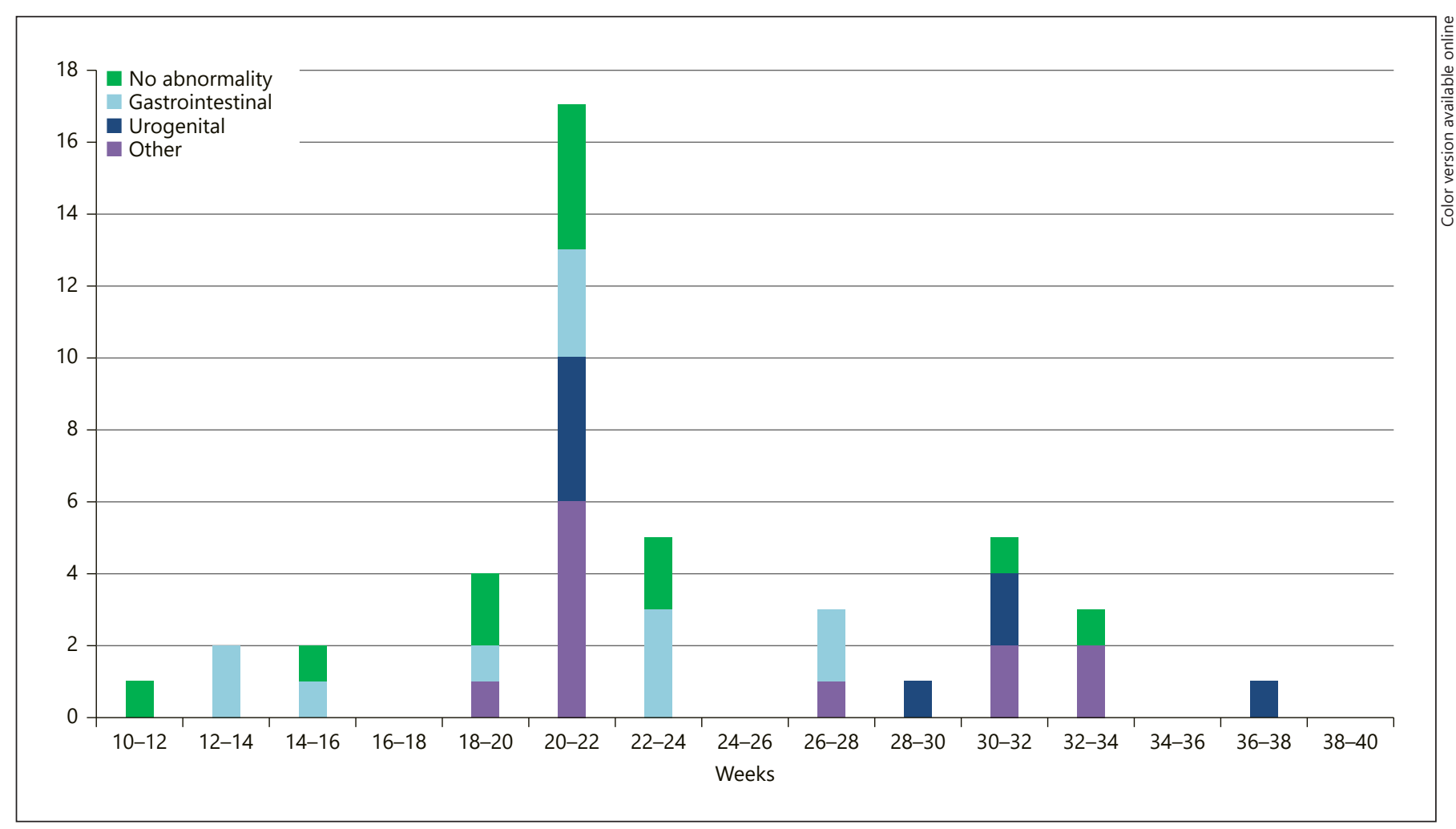

Fig. 4. The distribution of postnatal diagnoses according to gestational age at diagnosis in male fetuses.

$60.0 \%)$ when only the group with a postnatally confirmed abnormality, related to the intra-abdominal cyst, was taken into account.

Approximately one-third of the male neonates with a prenatally detected cyst $(n=15 / 44,34.1 \%)$ underwent surgery after birth; this is half of the male neonates where an abnormality related to the intra-abdominal cyst was confirmed after birth $(n=15 / 30,50.0 \%)$. The maximum prenatal size of cysts was not significantly different between males that had surgery and those who did not $(p=$ $0.54)$.

\section{Ovarian Cysts}

Nearly two-thirds $(81 / 114,71.1 \%)$ of the intra-abdominal cysts in females were of ovarian origin. Ovarian cysts were diagnosed at a significantly later gestational age compared with cysts of a different origin $(p<0.001)$. Most of the ovarian cysts regressed spontaneously in the pre- or postnatal period $(54 / 81,66.7 \%)$. An overview of these cases is provided in online supplementary Tables S1 and S2 (see www.karger.com/doi/10.1159/000495506). Spontaneous regression occurred significantly more often in cysts that had a maximum prenatal size smaller than 40 $\mathrm{mm}(39 / 47$ [83.0\%] vs. $15 / 34$ [44.1\%], $p<0.001)$ and in simple cysts (38/48 [79.2\%] vs. $17 / 33$ [51.5\%], $p=0.004)$. The regression rate was highest in cysts that were both simple and smaller than $40 \mathrm{~mm}(29 / 32,90.6 \%)$. None of the fetuses with an ovarian cyst showed ultrasonographic signs of anemia in the prenatal period, irrespective of the presence of a complicated cyst.

Most of the neonates with a persistent ovarian cyst ( $n=20 / 27,74.1 \%)$ underwent surgery. In $18(90.0 \%)$ of these neonates, adnexal torsion had occurred. Of the neonates who did not undergo surgery, in 4 cases, previous adnexal torsion was suspected based on the presence of calcifications and/or debris at postnatal ultrasound report. In 28 cases, normal ovaries were seen on postnatal ultrasound and in the other cases $(n=29)$, this was not clearly mentioned in the postnatal sonography reports. Torsion occurred significantly more often in ovarian cysts with a size $\geq 40 \mathrm{~mm}(11 / 18$ vs. $11 / 63, p=0.001)$ or complex cysts $(15 / 33$ vs. $7 / 48, p=0.008)$. Although this was less likely, torsion also occurred in 2 of 32 cases with simple cysts smaller than $40 \mathrm{~mm}(6.3 \%)$. 
Table 2. Postnatal diagnoses, according to affected organ system and gender, including the number of cases that underwent surgery

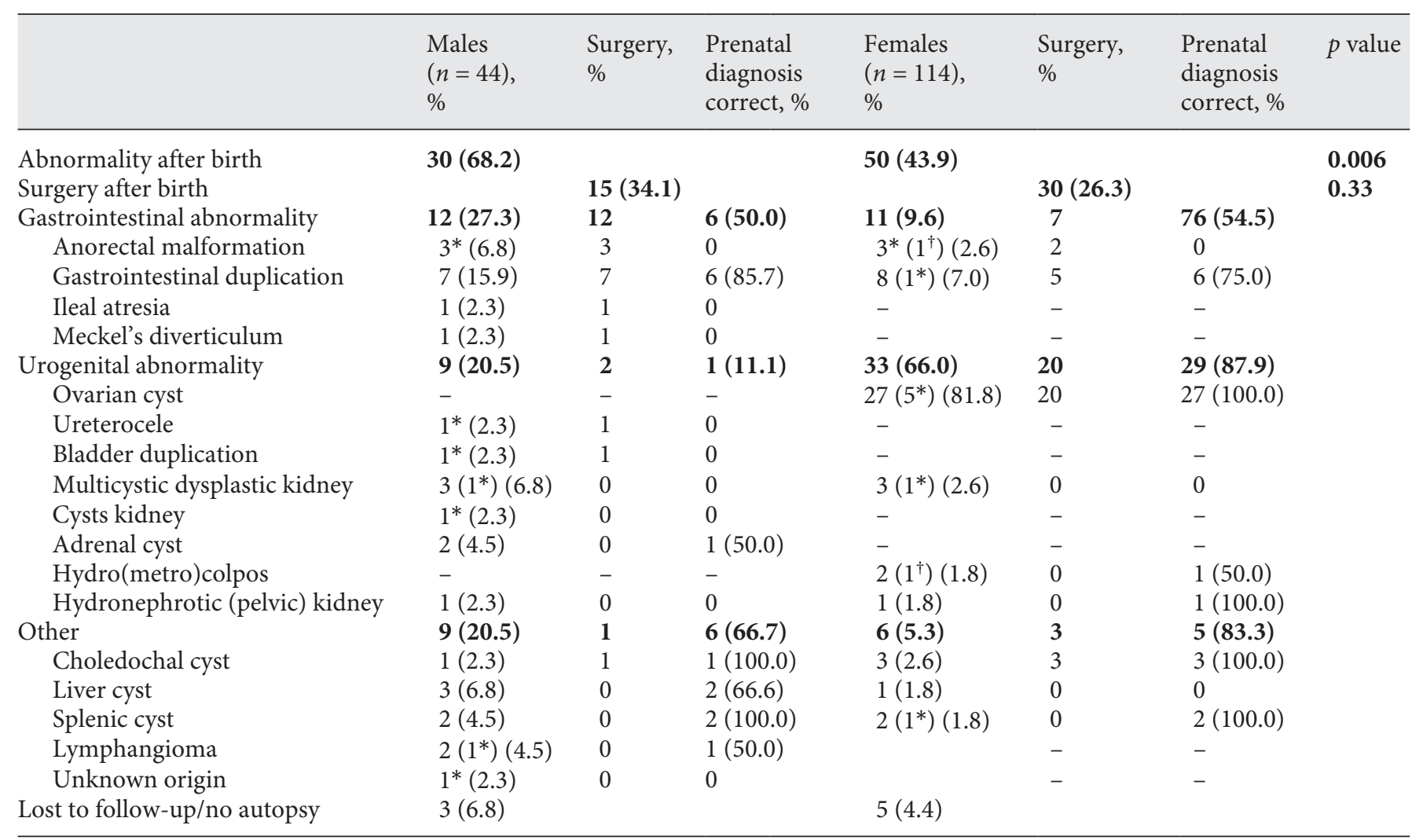

Ovarian cysts that spontaneously regressed were excluded $(n=54) .{ }^{*}$ Associated anomalies. ${ }^{\dagger}$ Neonatal death.

\section{Accuracy of Prenatal Diagnosis}

In most females $(40 / 50,80.0 \%)$, the postnatal diagnosis had been taken into consideration prenatally. Discrepancies were found in cases with an anorectal malformation, multicystic dysplastic kidney(s), hydro(metro)colpos, and liver cyst. The correct diagnosis was most frequently considered prenatally in cases with ovarian cysts, gastrointestinal duplications, choledochal cysts, and splenic cysts.

The correct diagnosis was considered prenatally in $13 / 30(43.3 \%)$ male fetuses. In 17 cases (56.7\%), a different diagnosis was made after birth. Anorectal malformations and abnormalities involving the kidneys were mostly not recognized prenatally. The highest similarity in prenatal and postnatal diagnosis in males was found in cases with gastrointestinal duplications, choledochal cyst, splenic cysts, and liver cysts. An overview of the number of correct diagnoses according to organ system is provided in Table 2 .

\section{Discussion}

In this study comparing prenatally detected intra-abdominal cysts in male and female fetuses on ultrasound, it was found that the cysts were more frequent and significantly larger in female fetuses. The gestational age at diagnosis of male fetuses compared with female fetuses was significantly lower. Significantly more males had postnatally confirmed abnormalities and associated anomalies. After exclusion of ovarian cysts, the maximum diameter, the gestational age at diagnosis and the proportion of cases with confirmed abnormalities and associated anomalies after birth were comparable between both genders.

Although the prevalence of anorectal malformations is low $(1: 5,000)$ [12], this anomaly was relatively common in the study population $(6: 158,3.8 \%)$. All but one had associated anomalies. The intra-abdominal cyst regressed in 1 of 3 females and 2 of 3 males. Furthermore, in the 2 males, the intra-abdominal cyst occurred in the first tri- 
mester. Similar cases were reported by Khalil et al. [5]. Careful evaluation of the fetal perianal muscular complex seems indicated in the presence of an intra-abdominal cyst, whereby it should be taken into account that this muscular complex can be visualized with $90-100 \%$ certainty between 23 and 34 weeks of gestation [13].

The spontaneous regression rate of ovarian cysts was high in this study (67.9\%), compared with Bascietto et al. (53.8\%) [14]. This could be caused by a higher proportion of smaller and simple cysts and the fact that in this study, regressing cysts that were diagnosed in the third trimester were classified as ovarian cyst, if this was considered the most likely diagnosis prenatally based on localization and ultrasound characteristics. Because of the natural history of fetal ovarian cysts [15], this seemed legitimate. We found, similar to previous research, a higher risk of torsion and need for surgery after birth in complex cysts and cysts $\geq 40 \mathrm{~mm}[14,16]$. In complex and/or large ovarian cysts, close prenatal surveillance is generally advised because of the risk of complications, such as bowel obstruction and polyhydramnios [17]. Within this cohort, a small number of cases developed polyhydramnios, but none resulted in a spontaneous preterm delivery. Because the presence of an ovarian cyst is usually not considered an indication for induction of labor [18] and severe hemorrhage resulting in anemia is extremely rare $[19,20]$, the need for stringent (e.g., weekly) prenatal follow-up in the third trimester in cases with large and/or complex ovarian cysts should be questioned. In cases with suspected ovarian cysts that are small and simple, have not changed in size or appearance, or regressed during pregnancy, parental reassurance seems appropriate.

In none of the cases, prenatal aspiration of ovarian cysts was performed. This intervention remains a subject of discussion, since randomized controlled trials comparing the outcome and risks of different fetal and neonatal management options are lacking $[14,21]$. The (possible) lower risk of ovarian torsion should be weighed against the risks of preterm labor, infection, and fetal injuries [16, $21]$. Based on the current evidence, prenatal aspiration might only be considered in exceptional cases, such as a large cyst that compresses bowel or leads to impaired ductus venosus flow [14].

After birth, unnecessary surgical intervention should be avoided, but early surgery could prevent complications such as torsion and thereby preserve ovarian tissue. In this study, most cases with ovarian torsion seem to have undergone surgery $(18 / 22)$ and the ovary could be preserved in just under half of the cases with adnexal torsion (7/18). However, the presence of normal appearing

Fetal Abdominal Cysts ovaries was not always described in the postnatal ultrasound reports. Because autoamputation following chronic adnexal torsion could not be excluded [22], the occurrence of torsion was classified as "unknown" in these cases.

In fetuses with a high risk of adnexal torsion (large and/or complex cysts), ultrasound shortly after birth is recommended. No prospective studies comparing longterm fertility outcome between conservative and surgical treatment are reported and are therefore required [16].

All male and nearly all female neonates with a gastrointestinal abnormality underwent surgery. The surgery rate on cysts originating from other organ systems was lower, except for choledochal cysts and ovarian cysts. The rate of surgery was similar to that reported in studies with comparable inclusion criteria $[3,7]$, but much lower than reported by Catania et al. [2] (81\%). This can be explained by the high proportion of cysts that were diagnosed in the late second and third trimester and few cases with spontaneous regression.

Chromosomal or genetic abnormalities were found only in fetuses with associated abnormalities. Based on these results, chromosomal analysis is not indicated in cases with isolated abdominal cysts. This corresponds with various studies in which fetal karyotype was investigated $[1,2,4,23]$.

The reported similarity between pre- and postnatal diagnoses ranges between 72.3 and $94.8 \%$ [1, 2, 4, 7]. The exclusion of obvious bowel obstructions and a larger percentage of complex abnormalities, such as anorectal malformations, might have resulted in a lower rate of similarity in the present study. Ovarian cysts were correctly diagnosed in the majority of cases, resulting in a higher similarity in females compared with males, although in most cases, multiple diagnoses were included in the differential diagnosis.

The strengths of the study are the large study population, the small number of cases that were lost to followup, and the fact that the results are presented according to fetal gender, which provides insight for prenatal counsellors. The limitations of the study are the retrospective design, the small number of cases per type of individual abnormalities, and the fact that the presence and morphology of ovaries was not always described in the postnatal ultrasound reports.

In summary, first-trimester cysts may be associated with anorectal malformations, particularly in male fetuses. Cysts appearing in the second trimester in females most likely originate from the gastrointestinal tract (bowel duplication), the kidneys, or bile duct, and the proba- 
bility of spontaneous regression is low. After 30 weeks of gestation, the most likely diagnosis in females is an ovarian cyst (with a high regression rate), but splenic cyst, gastrointestinal duplication, anorectal malformation, hydro(metro)colpos, and liver cysts should be included in the differential diagnosis. The differential diagnosis of cysts appearing in the second trimester in males should include gastrointestinal duplications, multicystic kidney dysplasia, and liver cysts. Cysts occurring in the third trimester in males are rare and do not seem to predominantly originate from one organ system.

These differences between males and females should be taken into account in prenatal counselling. In all fetuses with an intra-abdominal cyst, follow-up by ultrasound in the second and third trimester is recommended with evaluation of the perianal muscular complex, as well as a postnatal evaluation of the neonate, regardless of the gestational age at primary diagnosis. A multidisciplinary approach with pediatricians and pediatric surgeons to determine obstetric and neonatal management is strongly advised. Further research is required in order to determine prenatal and postnatal management in suspected ovarian cysts that are large and/or complex.

\section{Disclosure Statement}

The authors have no conflicts of interest to disclose. This study was undertaken without the support of funding.

\section{References}

1 Ozyuncu O, Canpolat FE, Ciftci AO, Yurdakok M, Onderoglu LS, Deren O. Perinatal outcomes of fetal abdominal cysts and comparison of prenatal and postnatal diagnoses. Fetal Diagn Ther. 2010;28(3):153-9.

2 Catania VD, Briganti V, Di Giacomo V, Miele V, Signore F, de Waure C, et al. Fetal intraabdominal cysts: accuracy and predictive value of prenatal ultrasound. J Matern Fetal Neonatal Med. 2016;29(10):1691-9.

3 Sherwood W, Boyd P, Lakhoo K. Postnatal outcome of antenatally diagnosed intra-abdominal cysts. Pediatr Surg Int. 2008 Jul; 24(7):763-5.

4 Marchitelli G, Stirnemann J, Acanfora MM, Rousseau V, Salomon LJ, Ville Y. Prenatal diagnosis of intra-abdominal cystic lesions by fetal ultrasonography: diagnostic agreement between prenatal and postnatal diagnosis. Prenat Diagn. 2015 Sep;35(9):848-52.

5 Khalil A, Cooke PC, Mantovani E, Bhide A, Papageorghiou AT, Thilaganathan B. Outcome of first-trimester fetal abdominal cysts: cohort study and review of the literature. Ultrasound Obstet Gynecol. 2014 Apr;43(4): 413-9.

6 Sepulveda W, Dickens K, Casasbuenas A, Gutierrez J, Dezerega V. Fetal abdominal cysts in the first trimester: prenatal detection and clinical significance. Ultrasound Obstet Gynecol. 2008 Dec;32(7):860-4.

7 Thakkar HS, Bradshaw C, Impey L, Lakhoo K. Post-natal outcomes of antenatally diagnosed intra-abdominal cysts: a 22-year single-institution series. Pediatr Surg Int. 2015 Feb;31(2): 187-90.
8 Akın MA, Akın L, Özbek S, Tireli G, Kavuncuoğlu S, Sander S, et al. Fetal-neonatal ovarian cysts-their monitoring and management: retrospective evaluation of 20 cases and review of the literature. J Clin Res Pediatr Endocrinol. 2010;2(1):28-33.

9 Heling KS, Chaoui R, Kirchmair F, Stadie S, Bollmann R. Fetal ovarian cysts: prenatal diagnosis, management and postnatal outcome. Ultrasound Obstet Gynecol. 2002 Jul;20(1): $47-50$.

10 Nussbaum AR, Sanders RC, Hartman DS, Dudgeon DL, Parmley TH. Neonatal ovarian cysts: sonographic-pathologic correlation. Radiology. 1988 Sep;168(3):817-21.

11 van der Cammen-van Zijp MH, Gischler SJ, Mazer P, van Dijk M, Tibboel D, Ijsselstijn H. Motor-function and exercise capacity in children with major anatomical congenital anomalies: an evaluation at 5 years of age. Early Hum Dev. 2010 Aug;86(8):523-8.

12 van den Hondel D, Sloots CE, Gischler SJ, Meeussen CJ, Wijnen RM, IJsselstijn H; Surgical Long-term Follow Up team. Prospective long-term follow up of children with anorectal malformation: growth and development until 5years of age. J Pediatr Surg. 2013 Apr; 48(4):818-25.

13 Moon MH, Cho JY, Kim JH, Min JY, Yang JH, Kim MY. In-utero development of the fetal anal sphincter. Ultrasound Obstet Gynecol. 2010 May;35(5):556-9.

14 Bascietto F, Liberati M, Marrone L, Khalil A, Pagani G, Gustapane S, et al. Outcome of ovarian cysts diagnosed on prenatal ultrasound: A systematic review and meta-analysis. Ultrasound Obstet Gynecol. 2017 Jul; 50(1):20-31.
15 Comparetto C, Giudici S, Coccia ME, Scarselli G, Borruto F. Fetal and neonatal ovarian cysts: what's their real meaning? Clin Exp Obstet Gynecol. 2005;32(2):123-5.

16 Monnery-Noché ME, Auber F, Jouannic JM, Bénifla JL, Carbonne B, Dommergues M, et al. Fetal and neonatal ovarian cysts: is surgery indicated? Prenat Diagn. 2008 Jan;28(1):15-20.

17 Bornstein E, Barnhard Y, Ferber A, Segarra P, Divon MY. Acute progression of a unilateral fetal ovarian cyst to complex bilateral cysts causing acute polyhydramnios. J Ultrasound Med. 2006 Apr;25(4):523-6.

18 Okumura M, Francisco RP, Shultz R, Zugaib M. Complex fetal ovarian cyst. J Ultrasound Med. 2015 May;34(5):925-6.

19 Vogtländer MF, Rijntjes-Jacobs EG, van den Hoonaard TL, Versteegh FG. Neonatal ovarian cysts. Acta Paediatr. 2003 Apr;92(4):498-501.

20 Vitezica I, Czernik C, Rothe K, Hinkson L, Ladendorf B, Henrich W. Prenatal diagnosis and management of a massive fetal ovarian hemorrhagic cyst torsion with secondary fetal anemia. J Clin Ultrasound. 2014 May;42(4): 219-22.

21 Tyraskis A, Bakalis S, David AL, Eaton S, De Coppi P. A systematic review and meta-analysis on fetal ovarian cysts: impact of size, appearance and prenatal aspiration. Prenat Diagn. 2017 Oct;37(10):951-8.

22 Focseneanu MA, Omurtag K, Ratts VS, Merritt DF. The auto-amputated adnexa: a review of findings in a pediatric population. J Pediatr Adolesc Gynecol. 2013 Dec;26(6):305-13.

23 Turgal M, Ozyuncu O, Yazicioglu A. Outcome of sonographically suspected fetal ovarian cysts. J Matern Fetal Neonatal Med. 2013 Nov;26(17):1728-32. 